Review Article

\title{
Protective Immunity against SARS Subunit Vaccine Candidates Based on Spike Protein: Lessons for Coronavirus Vaccine Development
}

\author{
Atin Khalaj-Hedayati \\ School of Biosciences, Faculty of Health and Medical Sciences, Taylor's University, Subang Jaya, Malaysia \\ Correspondence should be addressed to Atin Khalaj-Hedayati; atin.hedayati@gmail.com
}

Received 27 April 2020; Accepted 9 June 2020; Published 18 July 2020

Academic Editor: Nejat K. Egilmez

Copyright (c) 2020 Atin Khalaj-Hedayati. This is an open access article distributed under the Creative Commons Attribution License, which permits unrestricted use, distribution, and reproduction in any medium, provided the original work is properly cited.

\begin{abstract}
The recent outbreak of the novel coronavirus disease, COVID-19, has highlighted the threat that highly pathogenic coronaviruses have on global health security and the imminent need to design an effective vaccine for prevention purposes. Although several attempts have been made to develop vaccines against human coronavirus infections since the emergence of Severe Acute Respiratory Syndrome coronavirus (SARS-CoV) in 2003, there is no available licensed vaccine yet. A better understanding of previous coronavirus vaccine studies may help to design a vaccine for the newly emerged virus, SARS-CoV-2, that may also cover other pathogenic coronaviruses as a potentially universal vaccine. In general, coronavirus spike protein is the major antigen for the vaccine design as it can induce neutralizing antibodies and protective immunity. By considering the high genetic similarity between SARS-CoV and SARS-CoV-2, here, protective immunity against SARS-CoV spike subunit vaccine candidates in animal models has been reviewed to gain advances that can facilitate coronavirus vaccine development in the near future.
\end{abstract}

\section{Introduction}

Before 2003, coronaviruses were known to cause only common cold in humans, but currently, they are the reason for three outbreaks in the $21^{\text {st }}$ century: 2003 Severe Acute Respiratory Syndrome (SARS), 2012 Middle East Respiratory Syndrome (MERS), and 2019 Coronavirus Disease (COVID-19) [1]. Newly emerged virus, named SARS-CoV-2, was first discovered in December 2019, and in a short span of time, it has been announced as a global pandemic. On 2 June 2020, the virus spread has been noted in 213 countries and total confirmed cases climbed above 6.1 million with over 376000 deaths [2]. Despite numerous attempts to develop a vaccine against human coronavirus infection, there is no commercial vaccine available yet. Safety considerations and the degree of extensive diversity in antigenic variants are some of the potential reasons that limit coronavirus vaccine development [3].

Coronaviruses are enveloped positive-sense RNA virus, which are host-specific and can infect the human and a large number of animals [4]. Nucleotide substitution has been pro- posed to be one of the most important mechanisms of viral evolution in nature, and it is not necessarily surprising for an RNA virus that is a measurably evolving population over a short time, to have distinct variants $[5,6]$. Coronaviruses are phylogenetically classified into four major genera: Alphacoronavirus, Betacoronavirus, Gammacoronavirus, and Deltacoronavirus, and all three highly pathogenic coronaviruses for human belong to the Betacoronavirus genus. All epidemiological, pathophysiological, and immunological researches, which have been done on Betacoronaviruses may shed light on the understanding of SARS-CoV-2. This newly emerged virus is genetically more closer to SARS-CoV than MERS coronavirus with the existence of 380 amino acid substitution differences in the encoded proteins [7-9]. Therefore, previous advances made in developing SARS-CoV vaccines could be exploited for designing a vaccine not only for current COVID-19 pandemic but also for other highly pathogenic coronaviruses, so-called universal vaccine. This vaccine can be effective against all strains of the virus as a consequence of cross-protective immunity against conserved antigens. 
Moreover, the induced broad immunity can prevent the human from infection in the time of emerging a novel strain of the virus.

Inactivated virus and subunit vaccine technologies have been used to develop SARS-CoV vaccines. The inactive virus strategy is limited by safety considerations, as large quantities of the pathogenic virus are required directly in the vaccine preparation procedures. In contrast, the subunit vaccine that only relies on the antigen of interest by using recombinant technology is considered as a more reliable and safe technique. However, low immunogenicity might be a drawback in subunit vaccine development due to poor presentation to the immune system or incorrect folding of the antigens, but adjuvants can be involved in vaccination to boost immune responses and increase immunogenicity [10]. Alternatively, knowledge of the various viral proteins in inducing immune responses would facilitate subunit vaccine preparations [11]. The genome of coronaviruses includes a variable number of open reading frames that encode accessory proteins, nonstructural proteins, and structural proteins [12]. Most of the antigenic peptides are located in the structural proteins [13]. Spike surface glycoprotein (S), a small envelope protein (E), matrix protein $(\mathrm{M})$, and nucleocapsid protein $(\mathrm{N})$ are four main structural proteins. Since S-protein contributes to cell tropism and virus entry and also it is capable to induce neutralizing antibodies (NAb) and protective immunity, it is recognized as the most important target in coronavirus vaccine development among all other structural proteins [3, 14-17]. Moreover, amino acid sequence analysis has shown that S-protein contains conserved regions among the coronaviruses, which may be the basis for universal vaccine development $[18,19]$. This article reviewed the in vivo protective immunity of SARS-CoV S-protein vaccine candidates to provide an immunological evidence base that can aid coronavirus vaccine development in the future.

\section{Search Strategy and Selection Criteria}

References for this review were identified through searches of Scopus and PubMed for articles published between 2003 and 2020, using combinations of the terms "Severe Acute Respiratory Syndrome," SARS, SARS-CoV, vaccin*, immuniz*, immunis*, innocul*, develop*, design*, immunogenicity, and "immune response." The articles that indicated in vivo protective immunity study have been selected from the search result. The final reference list was generated based on this selection and relevant articles to the subtopics covered in this review, to highlight the immunological evidence base for coronavirus vaccine development and provide recommendations for navigating the limitations.

\section{Coronavirus Spike Protein: Promising Immunogen for Universal Vaccine Development}

The trimetric S-protein of coronaviruses is the reason for the crown shape of the viral particles, from which the name of the virus was given [20]. This structural protein belongs to class I viral fusion proteins and plays essential roles in the cell

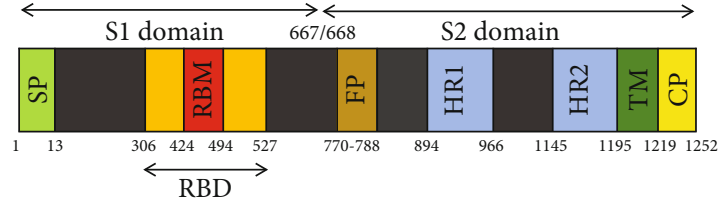

Figure 1: Schematic structure of the spike protein. Abbreviations: SP: signal peptide; RBD: receptor-binding domain; RMB: receptor motif binding; FP: fusion peptide; HR1: heptad repeat domain 1; HR2: heptad repeat domain 2; TM: transmembrane domain; CP: cytoplasmic domain. Numbers indicate amino acid sequence.

receptor binding, host tissue tropism and, pathogenesis. S-protein is cleaved at the S1/S2 site by host cell proteases during infection. Following cleavage, the protein is divided into an S1-ectodomain that recognizes a cognate cell surface receptor and an S2-membrane-anchored protein involved in viral entry [21]. The S1-protein contains a receptor-binding domain (RBD), which recognizes the angiotensin-converting enzyme 2 (ACE2) as one of the main receptors in the host [22]. $\mathrm{RBD}$ contains receptorbinding motif (RBM) that makes all the contacts with ACE2 and RBD core that is mostly conserved among coronaviruses $[21,23]$. Binding of the RBD to the ACE2 receptor provokes S2 conformational changes [24]. S2 contains membrane-anchoring, fusion peptide, and two heptad repeat domains (HR1 and HR2), which plays a key role in virus assembly and entry (Figure 1) [25-27]. The studies showed that vaccines based on S-protein can evoke the immune system to induce humoral and cellular responses and protect vaccinated animals from SARS viral challenges (Table 1) [17, 28-32].

In general, vaccine development for pathogens with a high rate of antigenic changes in their surface antigens such as SARS-CoV is difficult and complicated due to the emergence of vaccine escape variants over time. Hence, identification of conserved antigenic determinant on S-protein, which can induce cross-protective immune responses, may have implications in the development of an effective vaccine against all pathogenic coronaviruses. There is a limited number of SARS vaccine studies based on conserved antigens in which the potential protective immunity was not evaluated. For example, Zhang et al. identified a highly conserved antigenic determinant on S2 (amino acid 803 to 828) by using sera from convalescent SARS patients that could induce the S2-specific antibody with in vitro neutralizing activity against the SARS-CoV pseudovirus [33]. Other laboratory results indicated that peptides from the HR2 region of the S2 domain can block SARS-CoV infection in vitro $[34,35]$. However, the potential protection was not evaluated by challenge experiments, and further investigation is required to check in vivo neutralizing activity and also cross-protective immunity of these antigens for broad immune responses. Moreover, recent genome analysis disclosed that S2 and RBD core domains of SARS-CoV-2 are highly conserved, supporting the idea of universal vaccine design $[8,9,18$, 36-38]. It is notable that functional sites of S2 domain might be buried under $S 1$ in the native state of the virus structure, and it may affect the accessibility of the S2 antigens for the 


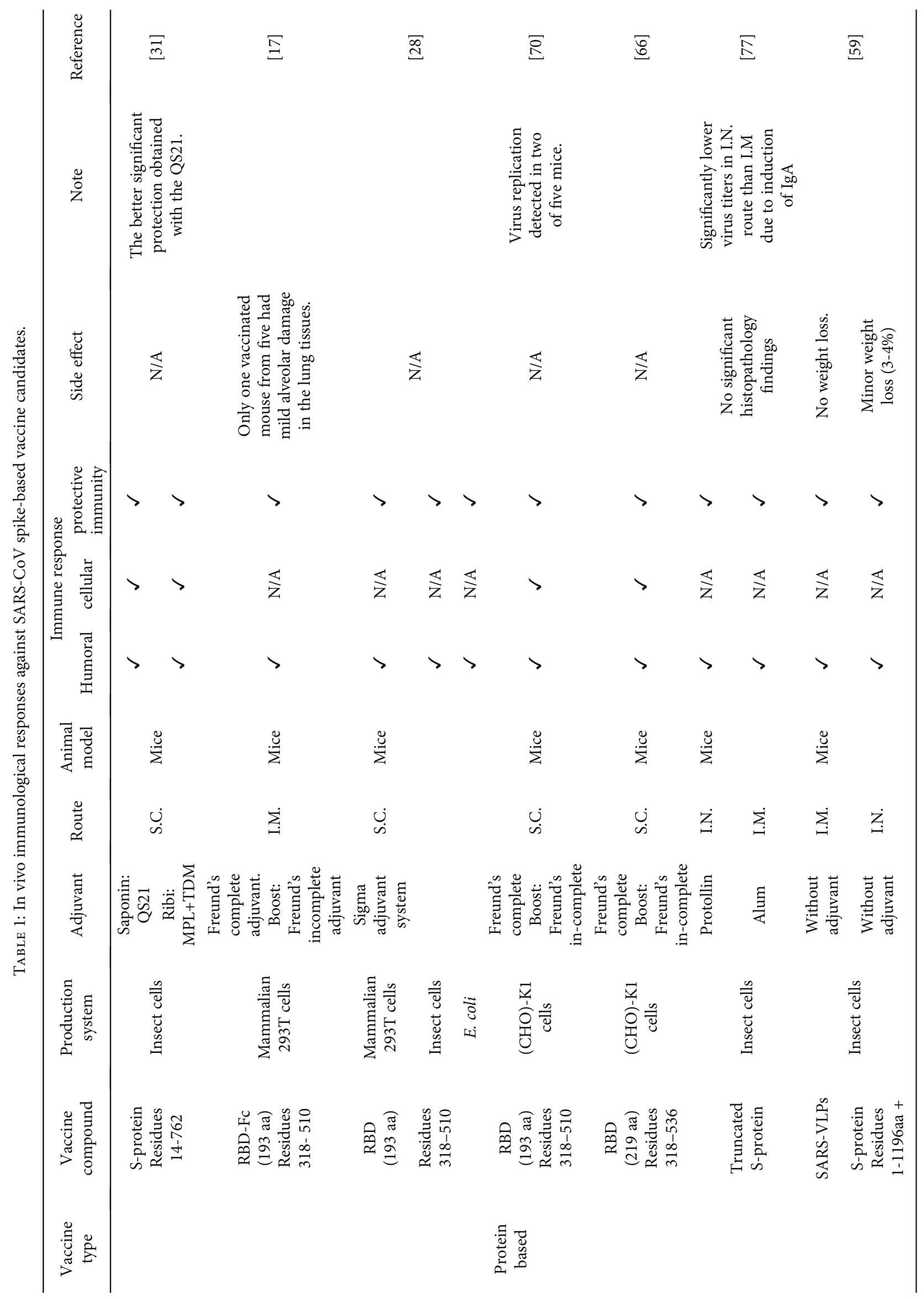




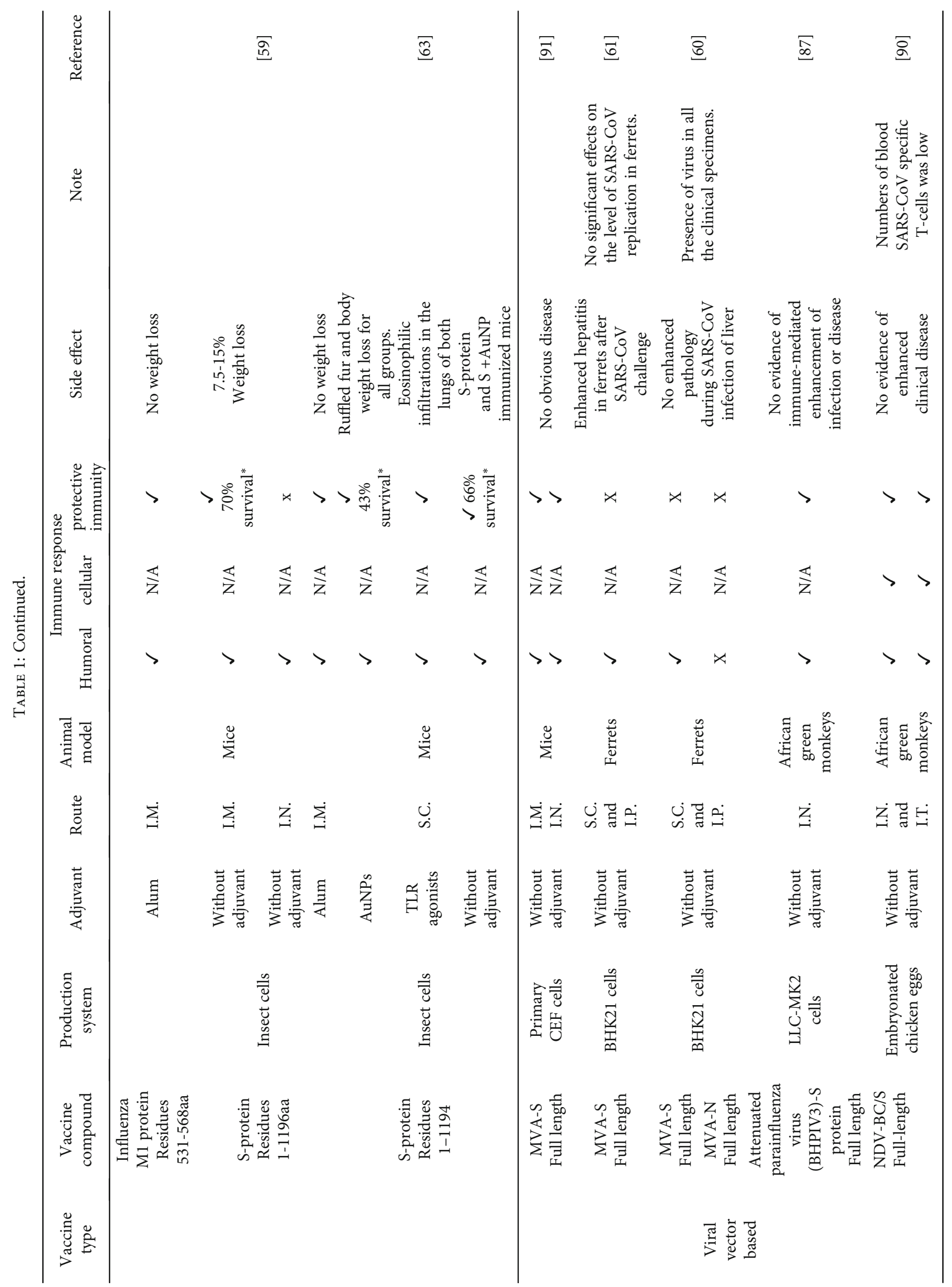




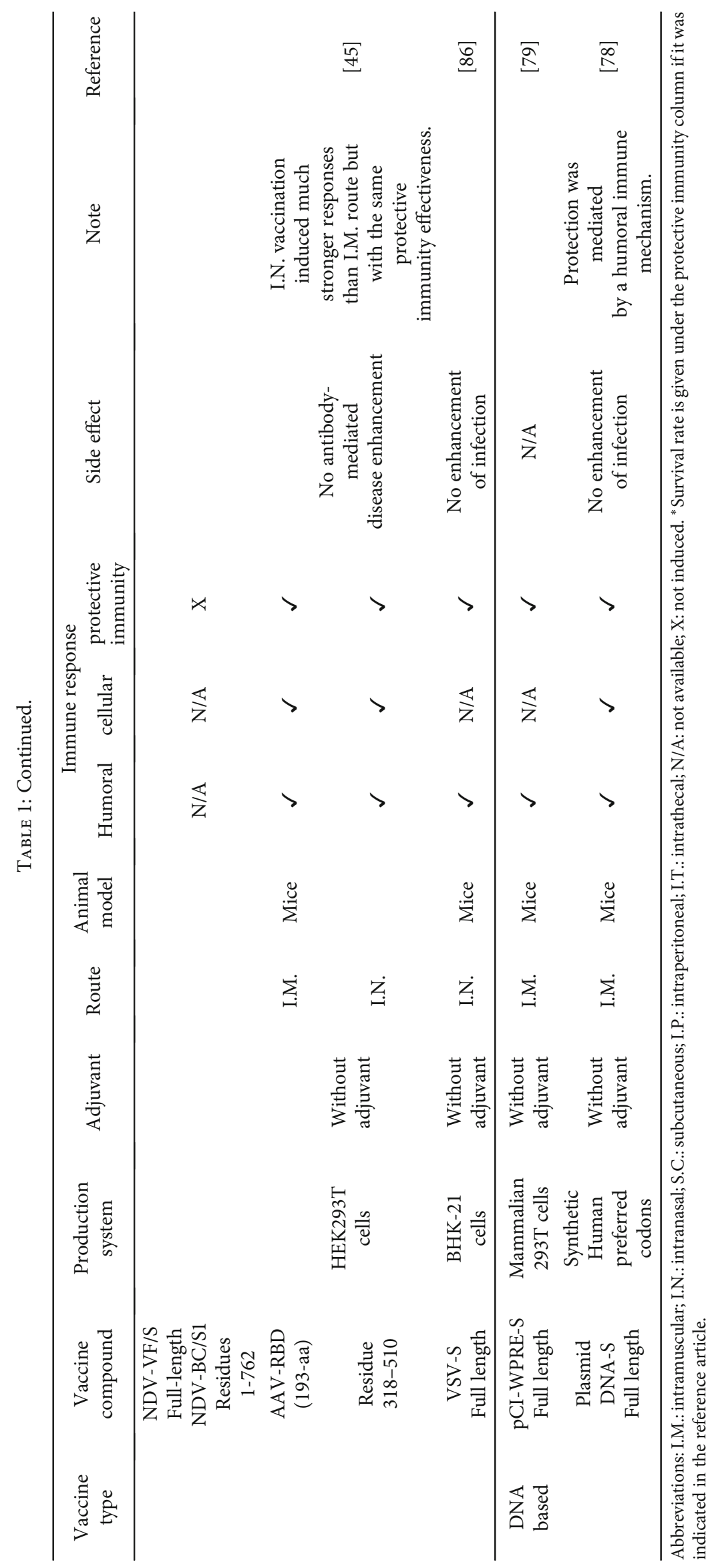


immune system but despite this shielding effect, $\mathrm{T}$ cell immune response to S2 domain that has been reported from fully recovered SARS patients suggests that it can be a candidate antigen for coronavirus vaccine development [39]. In addition, extra caution should be taken in designing vaccines that specifically target the S2 domain as disease severity can be augmented by elevating viral fusion to host cells at the early stage of the infection before activation of prior vaccine-induced immune responses $[40,41]$.

\section{What Immune Responses Are Required after Coronavirus Vaccination?}

Based on clinical studies, we can understand how the immune system of the patients reacts to coronavirus $[42,43]$. In general, the viral infection will be responded with humoral and cellular responses, which will be initiated by the innate immune system followed by the adaptive immune response. The latter consists of $\mathrm{B}$ cells that produce antibodies and $\mathrm{T}$ cells that kill virus-infected cells and both induce memory responses [44]. Generally, NAbs are the key factor for protecting human and animal models from coronavirus infections $[17,28,45]$. Sera of SARS patients indicate the presence of antibodies against SARS-CoV S, M, $\mathrm{E}$, and $\mathrm{N}$ proteins, but $\mathrm{NAb}$ is only induced by $\mathrm{S}$-protein [46]. Although anti-N antibody is present in SARS patient sera at a high-level and persisted for a long time (30 weeks after infection), recombinant $\mathrm{N}$-protein could not induce a detectable $\mathrm{NAb}$ in rabbit to neutralize the SARS-CoV infection [11]. Besides, deficient antibody production against S-protein in SARS-CoV infected patients with fatal outcomes has been reported that emphasizes the crucial role of the Sprotein NAb in SARS immunity [47]. Antibodies from the sera of 623 SARS patients were able to neutralize viruses containing S-protein from four different SARS-CoV strains, suggesting the potential cross-reactivity of these antibodies [48]. SARS-CoV-specific NAbs peak at four months and after that, gradually decline over time, indicating a reduction of the memory B cells against the virus [46, 49-51].

On the other hand, memory CD8 T cells can persist for at least six years in SARS patients who had recovered from the infection. These $\mathrm{T}$ cells are able to recognize and remove the infected cells in the lungs of patients. Whereas the memory B cell response and consequently NAbs are short-lived in SARS patients, generating long-lived memory $\mathrm{T}$ cell response is important, and it can be a complementary strategy in SARS vaccine design [46]. Interestingly, among the SARS-CoV CD8 $\mathrm{T}$ cell epitopes derived from different structural proteins, most peptides belong to S-protein [52]. In a clinical phase I study, a truncated S-protein DNA vaccine produced in bacterial cells induced SARS S-protein specific $\mathrm{T}$ cell response in all subjects and NAb responses detected in $80 \%$ of the individuals [53]. A DNA S-protein vaccine induced both CD4 and CD8 T cell responses, and S2 fragment encoding amino acid 681-980 elicited specific Cytotoxic T lymphocyte (CTL) response in animal models $[41,54]$. In another preclinical study, S-protein specific memory CD8 T cells could protect the mice against viral lethal challenge in the absence of NAbs, indicating that protection was elicited by memory CD8 T cells [55]. Also, dysregulated innate immune response that is a critical factor in the pathogenesis of SARS$\mathrm{CoV}$ can be negated by a potent $\mathrm{T}$ cell response [56]. Subsequently, the induction of cellular immune response is a goal for vaccine preparations as it plays an important role in antiviral immunity $[53,55,57,58]$.

Moreover, the studies suggested that mucosal immune responses represented by secretory IgA will also be important in the prevention of SARS-CoV infection [45]. Hence, intranasal (IN) immunization may be a preferable route to generate lung memory $\mathrm{T}$ cells and IgA specifically. However, IN immunization that produced local immunity in the upper respiratory tract but not in the lower tract where the virus replicates, performed less lung virus titer reduction than intramuscular (IM) route [59]. So, mucosal immune response localization should be considered while designing IN coronavirus vaccines. Eventually, future vaccines against coronaviruses should emphasize the generation of systematic and mucosal memory $\mathrm{T}$ cell responses and induction of longlived NAb against S-protein for optimal protection and virus clearance in the time of infection. Further, cross-protective immunity needs to be considered in the aim of coronavirus universal vaccine design.

\section{In Vivo Protective Immunity of SARS-CoV Spike-Based Vaccine Candidates}

In this article, recombinant S-protein-based vaccines, which include full-length or fragment vaccines, DNA-based vaccine, and viral vector-based vaccines that induce protective immunity in animal models have been reviewed (Table 1).

5.1. Protein-Based Vaccine. Full-length S-protein vaccines might be able to induce unwanted immune responses resulting in antibody-mediated disease enhancement (ADE) that can cause inflammatory and liver damage or enhancing infection after being challenged with SARS-CoV in animal models [60-63]. On the other hand, it has been demonstrated that fragments of S-protein, such as truncated S-protein or $\mathrm{RBD}$, have a great potential to be effective vaccine candidates against SARS with no evidence of harmful immune responses $[28,64,65]$. In several studies, Du and colleagues proposed SARS-CoV RBD as a fragment immunogen that can induce high titers of NAb and protective immunity in mice without immunopathological damages after being a challenge with the virus $[17,66,67]$. Administration of SARS-CoV recombinant S polypeptide (amino acids 14 to 762) elicited NAb in mice and protected the animals against upper and lower respiratory infection with SARS-CoV. This protein segment contains the $\mathrm{RBD}$ region as well as immunodominant and neutralizing epitopes [31, 68, 69].

To obtain highly expressed RBD economically and conveniently with biological functions, 193 amino acids of RBD (RBD-193) residues 318-510, have been expressed in different systems: mammalian cells 293T (RBD193-293T) and chinese hamster ovary K1 (RBD193-CHO-K1), insect cells Sf9 (RBD193-Sf9) and, E. coli (RBD193- Ec) [28, 70]. All RBD expressed proteins except RBD193-CHO-K1 induced complete protection against viral challenge in immunized mice. 
Virus replication was detected in two of five mice which were vaccinated with RBD193-CHO-K1. Interestingly, by adding 26 amino acids at the $\mathrm{C}$ terminal of RBD193 while expressed in the same CHO-K1 cell line (RBD219-CHO-K1), full protection against SARS-CoV challenge was observed due to high titers of RBD-specific antibody production. The extension may affect RBD structure into more immunogenic or more stable conformation, which could cause the induction of more potent protective antibodies in animal models compared to RBD193-CHO-K1. Cellular immune responses were also detected in RBD193-CHO-K1 and RBD219-CHO-K1 vaccinated mice $[28,70]$. Although the mammalian expressed RBD elicited stronger NAb responses than those expressed in insect and E. coli, Du and colleagues suggested any immunogens that elicited the serum NAb titers of $>1: 500$ in vaccinated mice would be effective enough for protection. So RBD193-Sf9 and RBD193-Ec were also considered as effective vaccines against viral challenge in mice with mean value $>1: 700$ for the serum NAb titers [28]. In general, insect and E. coli expression systems have lower production costs with a high productivity rate compared to the mammalian cell expression system, which make them more feasible for mass vaccine production [71, 72].

As the subunit vaccine may represent low immune responses, adjuvants or immunopotentiator can be used to increase immunogenicity by helping better presenting of immunogens to immune cells. The recombinant virus-like particles (VLP) have been shown to be an effective immunopotentiator and delivery system for foreign antigens in vaccine development. The repetitive antigen pattern on their surface can cause a stronger and broader induction of immune responses to the foreign antigen that is incorporated into the VLP [73, 74]. Liu et al. developed SARS S-protein VLP vaccine candidate with similar morphology and size to the SARS-CoV, and the IM immunization of the vaccine protected $100 \%$ of vaccinated mice from death and significantly reduced lung virus titer [59]. It is also important to concern about structural changes of immunogens that may reduce the immunogenicity after adjuvant implications in vaccine development. In one study, gold nanoparticles (AuNPs) containing S-protein showed weak protective ability against the SARS-CoV challenge compared to the control group because of the low induction of virus-specific IgG and NAb, which may be related to S-protein structural changes upon binding to AuNPs [63].

In the case of respiratory infections like SARS, both serum and lung immune responses are important, and it has been shown that relying on a systemic response may not be enough to reduce SARS-CoV infection in host [55, 75]. Mucosal immunization offers several advantages over other routes of antigen delivery, including convenience, cost-effectiveness, and induction of both local and systemic immune responses $[64,76]$. One study indicated that only IN immunization of mice by truncated S-protein with protollin adjuvant induced antigen-specific IgA responses in lung lavage fluid while both IN and IM administrations of the vaccine elicited comparable systemic responses. Serum collected IgG was able to possess strong protective activity against SARS-CoV, but the virus titer in the lung of mice was much lower for the IN vaccine compared to the IM route, and there was a qualitative correlation between the level of $\operatorname{IgA}$ and virus titer in the lung of animals. The fact that IM immunization developed a high level of serum antibodies but not detectable mucosal IgA with higher viral lung titer than IN route strongly indicates the essential role of the mucosal responses in SARS immunity [77].

5.2. DNA and Viral Vector-Based Vaccines. Numbers of DNA and viral vectored vaccines against SARS-CoV have been explored. A DNA vaccine that encodes full-length SARS Sprotein induced $\mathrm{NAb}$ and $\mathrm{T}$ cell responses and stimulated protective immunity [78]. Also, S-protein-based DNA vaccine encoding posttranscriptional enhancer (pCI-WPRE-S) not only improved immunogenicity in mice but also lessen DNA vaccine amount in vaccination. $10 \mu \mathrm{g}$ of the vaccine elicited NAb response equivalent to $25 \mu \mathrm{g}$ of Yang et al. codon-optimized vector $[78,79]$. The vaccine even at a dose as low as $2 \mu \mathrm{g}$ protects immunized animal against challenge infection [79]. This modification of DNA vaccine can minimize the risk of autoimmune responses or integration of the foreign DNA into the host genome [80]. The DNA Sprotein vaccine was able to elicit SARS-CoV-specific CD4 T cell responses in all tested individuals and CD8 $\mathrm{T}$ cell responses in $20 \%$ of subjects. Also, in prior clinical trials of DNA vaccines against HIV, Ebola, and West Nile virus, vaccine-specific $\mathrm{CD} 4 \mathrm{~T}$ cell responses were detected in nearly all subjects, while the frequency of measurable CD8 T cell responses varied from $7 \%$ to $64 \%$ [53, 81-83]. This aspect of DNA vaccine-induced immunity will require additional considerations while designing a DNA vaccine for coronaviruses.

The live viral vectored vaccines do not involve the complete pathogen, and they are qualified to induce mucosal humoral immune responses that may not be easily happened by DNA or protein vaccines $[84,85]$. A vesicular stomatitis virus (VSV) and an attenuated version of the human parainfluenza virus, a common respiratory pathogen in humans, both expressing the SARS-CoV S protein, were protective in animal models against SARS-CoV $[86,87]$. However, any viral vector vaccine based on common pathogens in the population would bring the concern regarding significant existence of prior immunity, which may restrict the replication of the viral vector in immunized models and decline the immunogenicity [88, 89]. Newcastle disease virus (NDV) is another viral vector that is antigenically distinct from a common human pathogen, and its natural host is birds. Remarkably, inoculation of the respiratory tract of African green monkeys (AGM) with recombinant NDV encoding SARS S-protein was protective enough against a high challenge dose of SARS-CoV. Vaccination with two constructs of NDV (NDV-VF/S or NDV-BC/S) resulted in a titer of serum NAb that was equaled or exceeded to parainfluenza construct immunization results $[87,90]$. Mice immunized with modified vaccinia virus Ankara that contains Sprotein (MVA-S) developed NAb and exhibited little or no replication of SARS CoV in the upper and lower respiratory tracts with no obvious disease after inoculation [91]. However, $\mathrm{NAb}$ responses in ferret against MVA-S were not protective in animals, but produce strong ADE effects $[60,61]$. 
As S-protein DNA-based vaccine, MVA-S, and a live SARS$\mathrm{CoV}$ demonstrated significant protective immunity in mice, different replication kinetics for SARS-CoV in mice and ferret can be the most likely cause for the difference in protective efficacy in these two animals $[61,78,91,92]$. Thus, extra caution should be taken in the proposed human trials of SARS vaccines due to the potential liver damage from immunization and virus infection.

The IN vaccination with adeno-associated virus (AAV) encoding SARS-RBD induced strong systemic humoral (IgG) and pulmonary humoral (IgA) responses with neutralizing activity as compared to IM vaccination. The immunization also protects $\mathrm{BALB} / \mathrm{c}$ mice against SARS-CoV infection. Surprisingly, AAV-RBD vaccination induced stronger CTL responses both in the lung and the spleen, and no sign of $\mathrm{ADE}$ in the animals was observed. Besides low systematic antibody responses in IN vaccination compared to the IM route, higher protection against virus challenge was achieved. The protective efficacy of AAV-RBD vaccination against SARS-CoV infection is correlated with the antibodies level, especially lung IgA [45]. Therefore, compared with the IM route, IN vaccination may fulfill multiple criteria for an effective and safe SARS-CoV vaccine.

\section{Conclusion}

The development of effective SARS-CoV-2 vaccine is crucial in aid of our public health preparedness against the current COVID-19 pandemic. Improved understanding of the protective immunity of SARS S-protein vaccine candidates would provide the immunological evidence base for future vaccine production. Moreover, this review reveals that despite the evidence of cross-reactivity of spike protein antibodies from SARS patients, there is a profound gap for immune response cross-protective evaluation against different strains of the virus. Now, it is time to direct research toward universal vaccine development by focusing on conserved immunogens that elicit potential cross-protective immunity to reduce the global threat of SARS-CoV-2 and any other pathogenic coronaviruses. Such studies would include preclinical experiments and also early phase clinical trials to assess the vaccine's immunogenicity. On the other hand, the previous vaccine studies of coronavirus have shown that immunization could often lead to adverse effect, such as ADE in animal models. Thus, it is important to clearly understand the potential risks of coronavirus vaccines and extra caution must be taken while testing the designed vaccines in human trials. In general, optimization of the vaccination regimen and evaluation of different vaccine strategies may be helpful to improve vaccine safety.

\section{Conflicts of Interest}

There is no conflict of interest.

\section{References}

[1] S. E. Reed, "The behavior of recent isolates of human respiratory coronavirus in vitro and in volunteers: evidence of hetero- geneity among 229E-related strains," Journal of Medical Virology, vol. 13, no. 2, pp. 179-192, 1984.

[2] Coronavirus disease 2019, World Heal Organ, 2020, https:// www.who.int/docs/default-source/coronaviruse/situationreports/20200602-covid-19-sitrep-134.pdf?sfvrsn=cc95e5d5_ 2.

[3] R. L. Graham, E. F. Donaldson, and R. S. Baric, "A decade after SARS: strategies for controlling emerging coronaviruses," Nature Reviews Microbiology, vol. 11, no. 12, pp. 836-848, 2013.

[4] S. R. Weiss and S. Navas-Martin, "Coronavirus pathogenesis and the emerging pathogen severe acute respiratory syndrome coronavirus," Microbiology and Molecular Biology Reviews, vol. 69, no. 4, pp. 635-664, 2005.

[5] A. S. Lauring and R. Andino, "Quasispecies theory and the behavior of RNA viruses," PLoS Pathogens, vol. 6, no. 7, article e1001005, 2010.

[6] D. Xu, Z. Zhang, and F. S. Wang, "SARS-associated coronavirus quasispecies in individual patients," The New England Journal of Medicine, vol. 350, no. 13, pp. 1366-1367, 2004.

[7] T. S. Fung and D. X. Liu, "Human coronavirus: host-pathogen interaction," Annual Review of Microbiology, vol. 73, no. 1, pp. 529-557, 2019.

[8] Y.-Z. Zhang and E. C. Holmes, "A genomic perspective on the origin and emergence of SARS-CoV-2," Cell, vol. 181, no. 2, pp. 223-227, 2020.

[9] A. Wu, Y. Peng, B. Huang et al., "Genome composition and divergence of the novel coronavirus $(2019-\mathrm{nCoV})$ originating in China," Cell Host \& Microbe, vol. 27, no. 3, pp. 325-328, 2020.

[10] A. W. Purcell, J. McCluskey, and J. Rossjohn, "More than one reason to rethink the use of peptides in vaccine design," Nature Reviews Drug Discovery, vol. 6, no. 5, pp. 404-414, 2007.

[11] M. Qiu, Y. Shi, Z. Guo et al., "Antibody responses to individual proteins of SARS coronavirus and their neutralization activities," Microbes and Infection, vol. 7, no. 5-6, pp. 882-889, 2005.

[12] Z. Song, Y. Xu, L. Bao et al., "From SARS to MERS, thrusting coronaviruses into the spotlight," Viruses, vol. 11, no. 1, p. 59, 2019.

[13] J. Cui, F. Li, and Z. L. Shi, "Origin and evolution of pathogenic coronaviruses," Nature Reviews Microbiology, vol. 17, no. 3, pp. 181-192, 2019.

[14] C. K. Li, H. Wu, H. Yan et al., "T cell responses to whole SARS coronavirus in humans," Journal of Immunology, vol. 181, no. 8, pp. 5490-5500, 2008.

[15] Y. He, J. Li, S. Heck, S. Lustigman, and S. Jiang, "Antigenic and immunogenic characterization of recombinant baculovirusexpressed severe acute respiratory syndrome coronavirus spike protein: implication for vaccine design," Journal of Virology, vol. 80, no. 12, pp. 5757-5767, 2006.

[16] G. Simmons, J. D. Reeves, A. J. Rennekamp, S. M. Amberg, A. J. Piefer, and P. Bates, "Characterization of severe acute respiratory syndrome-associated coronavirus (SARS-CoV) spike glycoprotein-mediated viral entry," Proceedings of the National Academy of Sciences of the United States of America, vol. 101, no. 12, pp. 4240-4245, 2004.

[17] L. Du, G. Zhao, Y. He et al., "Receptor-binding domain of SARS-CoV spike protein induces long-term protective immunity in an animal model," Vaccine, vol. 25, no. 15, pp. 2832 2838, 2007. 
[18] Y. Wan, J. Shang, R. Graham, R. S. Baric, and F. Li, "Receptor recognition by the novel coronavirus from Wuhan: an analysis based on decade-long structural studies of SARS Coronavirus," Journal of Virology, vol. 94, no. 7, pp. 1-9, 2020.

[19] P. A. Rota, M. S. Oberste, S. S. Monroe et al., "Characterization of a novel coronavirus associated with severe acute respiratory syndrome," Science, vol. 300, no. 5624, pp. 1394-1399, 2003.

[20] M. A. Marra, S. J. M. Jones, C. R. Astell et al., "The genome sequence of the SARS-associated coronavirus," Science, vol. 300, no. 5624, pp. 1399-1404, 2003.

[21] F. Li, "Structure, function, and evolution of coronavirus spike proteins," Annual Review of Virology, vol. 3, no. 1, pp. 237261, 2016.

[22] W. Li, M. J. Moore, N. Vasilieva et al., "Angiotensin-converting enzyme 2 is a functional receptor for the SARS coronavirus," Nature, vol. 426, no. 6965, pp. 450-454, 2003.

[23] F. Li, W. Li, M. Farzan, and S. C. Harrison, "Structure of SARS coronavirus spike receptor-binding domain complexed with receptor," Science, vol. 309, no. 5742, pp. 1864-1868, 2005.

[24] F. Li, M. Berardi, W. Li, M. Farzan, P. R. Dormitzer, and S. C. Harrison, "Conformational states of the severe acute respiratory syndrome coronavirus spike protein ectodomain," Journal of Virology, vol. 80, no. 14, pp. 6794-6800, 2006.

[25] T. M. Gallagher and M. J. Buchmeier, "Coronavirus spike proteins in viral entry and pathogenesis," Virology, vol. 279, no. 2, pp. 371-374, 2001.

[26] K. V. Holmes, "SARS-associated coronavirus," The New England Journal of Medicine, vol. 348, no. 20, pp. 1948-1951, 2003.

[27] B. Sainz, J. M. Rausch, W. R. Gallaher, R. F. Garry, and W. C. Wimley, "Identification and characterization of the putative fusion peptide of the severe acute respiratory syndromeassociated coronavirus spike protein," Journal of Virology, vol. 79, no. 11, pp. 7195-7206, 2005.

[28] L. Du, G. Zhao, C. C. S. Chan et al., "Recombinant receptorbinding domain of SARS-CoV spike protein expressed in mammalian, insect and E. coli cells elicits potent neutralizing antibody and protective immunity," Virology, vol. 393, no. 1, pp. 144-150, 2009.

[29] H. Hu, X. Lu, L. Tao et al., "Induction of specific immune responses by severe acute respiratory syndrome coronavirus spike DNA vaccine with or without interleukin-2 immunization using different vaccination routes in mice," Clinical and Vaccine Immunology, vol. 14, no. 7, pp. 894-901, 2007.

[30] Z. Chen, L. Zhang, C. Qin et al., "Recombinant modified vaccinia virus Ankara expressing the spike glycoprotein of severe acute respiratory syndrome coronavirus induces protective neutralizing antibodies primarily targeting the receptor binding region," Journal of Virology, vol. 79, no. 5, pp. 26782688, 2005.

[31] H. Bisht, A. Roberts, L. Vogel, K. Subbarao, and B. Moss, "Neutralizing antibody and protective immunity to SARS coronavirus infection of mice induced by a soluble recombinant polypeptide containing an N-terminal segment of the spike glycoprotein," Virology, vol. 334, no. 2, pp. 160-165, 2005.

[32] T. Zhou, H. Wang, D. Luo et al., "An exposed domain in the severe acute respiratory syndrome coronavirus spike protein induces neutralizing antibodies," Journal of Virology, vol. 78, no. 13, pp. 7217-7226, 2004.

[33] H. Zhang, G. Wang, J. Li et al., "Identification of an antigenic determinant on the S2 domain of the severe acute respiratory syndrome coronavirus spike glycoprotein capable of inducing neutralizing antibodies," Journal of Virology, vol. 78, no. 13, pp. 6938-6945, 2004.

[34] J. Zhu, G. Xiao, Y. Xu et al., "Following the rule: formation of the 6-helix bundle of the fusion core from severe acute respiratory syndrome coronavirus spike protein and identification of potent peptide inhibitors," Biochemical and Biophysical Research Communications, vol. 319, no. 1, pp. 283-288, 2004.

[35] C.-T. Keng, A. Zhang, S. Shen et al., "Amino acids 1055 to 1192 in the S2 region of severe acute respiratory syndrome coronavirus $S$ protein induce neutralizing antibodies: implications for the development of vaccines and antiviral agents," Journal of Virology, vol. 79, no. 6, pp. 3289-3296, 2005.

[36] A. C. Walls, Y. J. Park, M. A. Tortorici, A. Wall, A. T. McGuire, and D. Veesler, "Structure, function, and antigenicity of the SARS-CoV-2 spike glycoprotein," Cell, vol. 181, no. 2, pp. 281-292.e6, 2020.

[37] J. F.-W. Chan, K. H. Kok, Z. Zhu et al., "Genomic characterization of the 2019 novel human-pathogenic coronavirus isolated from a patient with atypical pneumonia after visiting Wuhan," Emerging Microbes \& Infections, vol. 9, no. 1, pp. 221-236, 2020.

[38] Y. S. Malik, S. Sircar, S. Bhat et al., "Emerging novel coronavirus (2019-nCoV) - current scenario, evolutionary perspective based on genome analysis and recent developments," Veterinary Quarterly, vol. 40, no. 1, pp. 68-76, 2020.

[39] Y.-D. Wang, W. Y. F. Sin, G. B. Xu et al., “T-cell epitopes in severe acute respiratory syndrome (SARS) coronavirus spike protein elicit a specific T-Cell immune response in patients who recover from SARS," Journal of Virology, vol. 78, no. 11, pp. 5612-5618, 2004.

[40] S. Khurana, C. L. Loving, J. Manischewitz et al., "Vaccineinduced anti-HA2 antibodies promote virus fusion and enhance influenza virus respiratory disease," Science Translational Medicine, vol. 5, no. 200, article 200ra114, 2013.

[41] Y. Guo, S. Sun, K. Wang, S. Zhang, W. Zhu, and Z. Chen, "Elicitation of immunity in mice after immunization with the S2 subunit of the severe acute respiratory syndrome coronavirus," DNA and Cell Biology, vol. 24, no. 8, pp. 510-515, 2005.

[42] G. Li, X. Chen, and A. Xu, "Profile of specific antibodies to the SARS-associated coronavirus," The New England Journal of Medicine, vol. 349, no. 5, pp. 508-509, 2003.

[43] D. Corti, J. Zhao, M. Pedotti et al., "Prophylactic and postexposure efficacy of a potent human monoclonal antibody against MERS coronavirus," Proceedings of the National Academy of Sciences of the United States of America, vol. 112, no. 33, pp. 10473-10478, 2015.

[44] D. D. Chaplin, "Overview of the immune response," Journal of Allergy and Clinical Immunology, vol. 125, no. 2, pp. S3-23, 2010.

[45] L. Du, G. Zhao, Y. Lin et al., "Intranasal vaccination of recombinant adeno-associated virus encoding receptor-binding domain of severe acute respiratory syndrome coronavirus (SARS-CoV) spike protein induces strong mucosal immune responses and provides long-term protection against SARSCoV infection," Journal of Immunology, vol. 180, no. 2, pp. 948-956, 2008.

[46] J. Chen and K. Subbarao, "The immunobiology of SARS," Annual Review of Immunology, vol. 25, no. 1, pp. 443-472, 2007.

[47] M. J. Cameron, L. Ran, L. Xu et al., "Interferon-mediated immunopathological events are associated with atypical innate 
and adaptive immune responses in patients with severe acute respiratory syndrome," Journal of Virology, vol. 81, no. 16, pp. 8692-8706, 2007.

[48] Y. Nie, G. Wang, X. Shi et al., "Neutralizing antibodies in patients with severe acute respiratory syndrome-associated coronavirus infection," The Journal of Infectious Diseases, vol. 190, no. 6, pp. 1119-1126, 2004.

[49] P. C. Y. Woo, S. K. P. Lau, B. H. L. Wong et al., "Longitudinal profile of immunoglobulin G (IgG), IgM, and IgA antibodies against the severe acute respiratory syndrome (SARS) coronavirus nucleocapsid protein in patients with pneumonia due to the SARS coronavirus," Clinical and Diagnostic Laboratory Immunology, vol. 11, no. 4, pp. 665-668, 2004.

[50] L. P. Wu, N. C. Wang, Y. H. Chang et al., "Duration of antibody responses after severe acute respiratory syndrome," Emerging Infectious Diseases, vol. 13, no. 10, pp. 1562-1564, 2007.

[51] F. Tang, Y. Quan, Z.-T. Xin et al., "Lack of peripheral memory $B$ cell responses in recovered patients with severe acute respiratory syndrome: a six-year follow-up study," Journal of Immunology, vol. 186, no. 12, pp. 7264-7268, 2011.

[52] W. J. Liu, M. Zhao, K. Liu et al., “T-cell immunity of SARSCoV: implications for vaccine development against MERSCoV," Antiviral Research, vol. 137, pp. 82-92, 2017.

[53] J. E. Martin, M. K. Louder, L. S. A. Holman et al., “A SARS DNA vaccine induces neutralizing antibody and cellular immune responses in healthy adults in a phase I clinical trial," Vaccine, vol. 26, no. 50, pp. 6338-6343, 2008.

[54] J. Huang, Y. Cao, J. Du, X. Bu, R. Ma, and C. Wu, "Priming with SARS CoV S DNA and boosting with SARS CoV S epitopes specific for $\mathrm{CD} 4+$ and $\mathrm{CD} 8+\mathrm{T}$ cells promote cellular immune responses," Vaccine, vol. 25, no. 39-40, pp. 6981-6991, 2007.

[55] R. Channappanavar, C. Fett, J. Zhao, D. K. Meyerholz, and S. Perlman, "Virus-specific memory CD8 T cells provide substantial protection from lethal severe acute respiratory syndrome coronavirus infection," Journal of Virology, vol. 88, no. 19, pp. 11034-11044, 2014.

[56] J. Zhao, J. Zhao, and S. Perlman, "T cell responses are required for protection from clinical disease and for virus clearance in severe acute respiratory syndrome coronavirus-infected mice," Journal of Virology, vol. 84, no. 18, pp. 9318-9325, 2010.

[57] Y. Z. Chen, G. Liu, S. Senju et al., "Identification of SARS-COV spike protein-derived and HLA-A2-restricted human CTL epitopes by using a new muramyl dipeptide-derivative adjuvant," International Journal of Immunopathology and Pharmacology, vol. 23, no. 1, pp. 165-177, 2010.

[58] T.-M. Fu, S. A. Dubey, D. V. Mehrotra et al., "Evaluation of cellular immune responses in subjects chronically infected with HIV type 1," AIDS Research and Human Retroviruses, vol. 23, no. 1, pp. 67-76, 2007.

[59] Y. V. Liu, M. J. Massare, D. L. Barnard et al., "Chimeric severe acute respiratory syndrome coronavirus (SARS-CoV) S glycoprotein and influenza matrix 1 efficiently form virus-like particles (VLPs) that protect mice against challenge with SARSCoV," Vaccine, vol. 29, no. 38, pp. 6606-6613, 2011.

[60] M. Czub, H. Weingartl, S. Czub, R. He, and J. Cao, "Evaluation of modified vaccinia virus Ankara based recombinant SARS vaccine in ferrets," Vaccine, vol. 23, no. 17-18, pp. 22732279, 2005.

[61] H. Weingartl, M. Czub, S. Czub et al., "Immunization with modified vaccinia virus Ankara-based recombinant vaccine against severe acute respiratory syndrome is associated with enhanced hepatitis in ferrets," Journal of Virology, vol. 78, no. 22, pp. 12672-12676, 2004.

[62] Y. Honda-Okubo, D. Barnard, C. H. Ong, B. H. Peng, C. T. K. Tseng, and N. Petrovsky, "Severe acute respiratory syndromeassociated coronavirus vaccines formulated with delta inulin adjuvants provide enhanced protection while ameliorating lung eosinophilic immunopathology," Journal of Virology, vol. 89, no. 6, pp. 2995-3007, 2015.

[63] H. Sekimukai, N. Iwata-Yoshikawa, S. Fukushi et al., "Gold nanoparticle-adjuvanted $\mathrm{S}$ protein induces a strong antigenspecific IgG response against severe acute respiratory syndrome-related coronavirus infection but fails to induce protective antibodies and limit eosinophilic infiltration in lungs," Microbiology and Immunology, vol. 64, no. 1, pp. 3351, 2019.

[64] J.-S. Lee, H. Poo, D. P. Han et al., "Mucosal immunization with surface-displayed severe acute respiratory syndrome coronavirus spike protein on Lactobacillus casei induces neutralizing antibodies in mice," Journal of Virology, vol. 80, no. 8, pp. 4079-4087, 2006.

[65] Z. Zhou, P. Post, R. Chubet et al., "A recombinant baculovirusexpressed $\mathrm{S}$ glycoprotein vaccine elicits high titers of SARSassociated coronavirus (SARS-CoV) neutralizing antibodies in mice," Vaccine, vol. 24, no. 17, pp. 3624-3631, 2006.

[66] L. Du, G. Zhao, C. C. S. Chan et al., "A 219-mer CHOexpressing receptor-binding domain of SARS-CoV S protein induces potent immune responses and protective immunity," Viral Immunology, vol. 23, no. 2, pp. 211-219, 2010.

[67] Y. He, H. Lu, P. Siddiqui, Y. Zhou, and S. Jiang, "Receptor-binding domain of severe acute respiratory syndrome coronavirus spike protein contains multiple conformationdependent epitopes that induce highly potent neutralizing antibodies," Journal of Immunology, vol. 174, no. 8, pp. 4908-4915, 2005.

[68] G. J. Babcock, D. J. Esshaki, W. D. Thomas, and D. M. Ambrosino, "Amino acids 270 to 510 of the severe acute respiratory syndrome coronavirus spike protein are required for interaction with receptor," Journal of Virology, vol. 78, no. 9, pp. 4552-4560, 2004.

[69] Y. He, Y. Zhou, H. Wu et al., "Identification of immunodominant sites on the spike protein of severe acute respiratory syndrome (SARS) coronavirus: implication for developing SARS diagnostics and vaccines," Journal of Immunology, vol. 173, no. 6, pp. 4050-4057, 2004.

[70] L. Du, G. Zhao, L. Li et al., "Antigenicity and immunogenicity of SARS-CoV S protein receptor-binding domain stably expressed in CHO cells," Biochemical and Biophysical Research Communications, vol. 384, no. 4, pp. 486-490, 2009.

[71] C. S. Boosani and A. Sudhakar, "Cloning, purification, and characterization of a non-collagenous anti- angiogenic protein domain from human $\alpha 1$ type IV collagen expressed in $S f 9$ cells," Protein Expression and Purification, vol. 49, no. 2, pp. 211-218, 2006.

[72] H. Saitoh, J. Uwada, and K. Azusa, "Strategies for the Expression of SUMO-Modified Target Proteins in Escherichia coli," in BT - SUMO Protocols, H. D. Ulrich, Ed., pp. 211-221, Humana Press, Totowa, NJ, USA, 2009.

[73] R. A. Bright, D. M. Carter, S. Daniluk et al., "Influenza viruslike particles elicit broader immune responses than whole virion inactivated influenza virus or recombinant hemagglutinin," Vaccine, vol. 25, no. 19, pp. 3871-3878, 2007. 
[74] H. Song, V. Wittman, A. Byers et al., "In vitro stimulation of human influenza-specific CD8+ T cells by dendritic cells pulsed with an influenza virus-like particle (VLP) vaccine," Vaccine, vol. 28, no. 34, pp. 5524-5532, 2010.

[75] C. Ma, Y. Li, L. Wang et al., "Intranasal vaccination with recombinant receptor-binding domain of MERS-CoV spike protein induces much stronger local mucosal immune responses than subcutaneous immunization: implication for designing novel mucosal MERS vaccines," Vaccine, vol. 32, no. 18, pp. 2100-2108, 2014.

[76] J. F. M. L. Seegers, "Lactobacilli as live vaccine delivery vectors: progress and prospects," Trends in Biotechnology, vol. 20, no. 12 , pp. 508-515, 2002.

[77] M. C. Hu, T. Jones, R. T. Kenney, D. L. Barnard, D. S. Burt, and G. H. Lowell, "Intranasal protollin-formulated recombinant SARS S-protein elicits respiratory and serum neutralizing antibodies and protection in mice," Vaccine, vol. 25, no. 34, pp. 6334-6340, 2007.

[78] Z. Y. Yang, W. P. Kong, Y. Huang et al., "A DNA vaccine induces SARS coronavirus neutralization and protective immunity in mice," Nature, vol. 428, no. 6982, pp. 561-564, 2004.

[79] B. Callendret, V. Lorin, P. Charneau et al., "Heterologous viral RNA export elements improve expression of severe acute respiratory syndrome (SARS) coronavirus spike protein and protective efficacy of DNA vaccines against SARS," Virology, vol. 363, no. 2, pp. 288-302, 2007.

[80] Z. Cui, "DNA vaccine," Advances in Genetics, vol. 54, pp. 257289, 2005.

[81] A. T. Catanzaro, M. Roederer, R. A. Koup et al., "Phase I clinical evaluation of a six-plasmid multiclade HIV-1 DNA candidate vaccine," Vaccine, vol. 25, no. 20, pp. 4085-4092, 2007.

[82] J. E. Martin, N. J. Sullivan, M. E. Enama et al., "A DNA vaccine for Ebola virus is safe and immunogenic in a phase I clinical trial," Clinical and Vaccine Immunology, vol. 13, no. 11, pp. 1267-1277, 2006.

[83] J. E. Martin, T. C. Pierson, S. Hubka et al., "A West Nile virus DNA vaccine induces neutralizing antibody in healthy adults during a phase 1 clinical trial," The Journal of Infectious Diseases, vol. 196, no. 12, pp. 1732-1740, 2007.

[84] S. Crotty, B. L. Lohman, F. X. Lü, S. Tang, C. J. Miller, and R. Andino, "Mucosal immunization of cynomolgus macaques with two serotypes of live poliovirus vectors expressing simian immunodeficiency virus antigens: stimulation of humoral, mucosal, and cellular immunity," Journal of Virology, vol. 73, no. 11, pp. 9485-9495, 1999.

[85] D. Wang, E. R. Kandimalla, D. Yu, J. X. Tang, and S. Agrawal, "Oral administration of second-generation immunomodulatory oligonucleotides induces mucosal Th1 immune responses and adjuvant activity," Vaccine, vol. 23, no. 20, pp. 2614-2622, 2005.

[86] S. U. Kapadia, J. K. Rose, E. Lamirande, L. Vogel, K. Subbarao, and A. Roberts, "Long-term protection from SARS coronavirus infection conferred by a single immunization with an attenuated VSV-based vaccine," Virology, vol. 340 , no. 2, pp. 174-182, 2005.

[87] A. Bukreyev, E. W. Lamirande, U. J. Buchholz, L. N. Vogel, W. R. Elkins, M. St Claire et al., "Mucosal immunisation of African green monkeys (Cercopithecus aethiops) with an attenuated parainfluenza virus expressing the SARS coronavirus spike protein for the prevention of SARS," The Lancet, vol. 363, no. 9427, pp. 2122-2127, 2004.
[88] Y. Zhi, J. Figueredo, G. P. Kobinger et al., "Efficacy of severe acute respiratory syndrome vaccine based on a nonhuman primate adenovirus in the presence of immunity against human adenovirus," Human Gene Therapy, vol. 17, no. 5, pp. 500506, 2006.

[89] N. Kanesa-thasan, J. J. Smucny, C. H. Hoke Jr. et al., "Safety and immunogenicity of NYVAC-JEV and ALVAC-JEV attenuated recombinant Japanese encephalitis virus - poxvirus vaccines in vaccinia-nonimmune and vaccinia-immune humans," Vaccine, vol. 19, no. 4-5, pp. 483-491, 2000.

[90] J. M. DiNapoli, A. Kotelkin, L. Yang et al., "Newcastle disease virus, a host range-restricted virus, as a vaccine vector for intranasal immunization against emerging pathogens," Proceedings of the National Academy of Sciences of the United States of America, vol. 104, no. 23, pp. 9788-9793, 2007.

[91] H. Bisht, A. Roberts, L. Vogel et al., "Severe acute respiratory syndrome coronavirus spike protein expressed by attenuated vaccinia virus protectively immunizes mice," Proceedings of the National Academy of Sciences of the United States of America, vol. 101, no. 17, pp. 6641-6646, 2004.

[92] K. Subbarao, J. McAuliffe, L. Vogel et al., "Prior infection and passive transfer of neutralizing antibody prevent replication of severe acute respiratory syndrome coronavirus in the respiratory tract of mice," Journal of Virology, vol. 78, no. 7, pp. 3572-3577, 2004. 